\title{
Maximum Likelihood DOA Estimation Based on the Cross-Entropy Method
}

\author{
Yen-Chih Chen and Yu T. Su \\ Department of Communications Engineering \\ National Chiao Tung University \\ 1001 Ta Hsueh Road, Hsinchu 30056, TAIWAN \\ joechen.cm88g@nctu.edu.tw,ytsu@ mail.nctu.edu.tw
}

\begin{abstract}
In this paper, we propose two simulation based maximum likelihood (ML) methods to estimate the direction of arrival (DOA) by a novel combination of the Cross-Entropy (CE) method and the polynomial parameterization scheme. The CE method is an efficient stochastic approximation method for solving both discrete and continuous optimization problems. We bridge the ML approach and the stochastic search algorithm by properly randomizing the desired parameters. Numerical results show that the proposed CE-based algorithms yield highly accurate DOA estimation with fast convergence rate while requiring only linear processing complexity. Compared with the conventional iterative quadratic maximization likelihood (IQML) method, the proposed algorithms can alleviate the error propagation effect in low signal to noise ratio (SNR) region and asymptotically approach the Cramér-Rao bound in high SNR region.
\end{abstract}

\section{INTRODUCTION}

The problem of DOA estimation has been extensively investigated in the last few decades [1]. Among various DOA estimation approaches, the class of maximum likelihood (ML) methods is both unbiased and asymptotically efficient. Moreover, the ML methods can alleviate the mutual cancellation effect caused by the coherent sources and provide high estimation accuracy. However, the multi-dimensional optimization nature of the ML methods also require high computing complexity. Bresler and Macovski [2] suggested a polynomial parameterization approach for conditional ML estimation and applied iterative projection to reduce the computation burden. The resulting ML algorithm, abbreviated IQML, is an efficient and near-optimal solution. However, IQML suffers from slow convergence rate in low SNR region and it does not give satisfactory performance when the sample size is small.

In this paper, we suggest two novel simulated-based optimization approach to solve the ML DOA estimation problem. The Cross-Entropy (CE) method developed by Rubinstein [3] was originally aimed to solve the rare event simulation problem. It was later proposed as an unified approach to perform global search for combinatorial and continuous multiextremal optimization problems. Under the CE method framework, deterministic signals or parameters, e.g. DOA in array signal processing, are randomized according to some para- metric distributions related with a set of dynamic parameters. Through iteratively minimizing the Kullback-Leibler distance (cross entropy) between the the parametric distribution and the target distribution, we can update the set of parameters and improved the estimate quality. Thanks to the accurate and robust nature of the $\mathrm{CE}$ method [3], we are able to obtain highly accurate ML DOA estimation and eliminate the error propagation effect incurred by the iterative projection in the conventional IQML algorithm.

To solve the complexity issue associated with the simulated searching approach, we approximate source information by sample covariance matrix and adopt the polynomial parameterization procedure used in the IQML algorithm to translate the original problem into one that is a computational much more efficient. In short, this paper presents a new estimate method that not only links the conventional conditional ML and the simulated approximation approaches but also suggest a novel bridging procedure so that the advantages of both approaches are preserved.

The organization of this paper is as follows. In Section II, we describe the system model of array signal processing and review the conventional IQML method. In Section III, we describe the generic concept of the CE method. In Section IV, we present the proposed maximum likelihood estimation based on the CE method. In Section V, a polynomial parameterizing $\mathrm{CE}$ method is proposed to lower the computation complexity. In Section VI, we provide some simulation examples and discuss the numerical behavior. Finally, in the last Section, we give summary remarks on the proposed new methods and suggest some future works.

\section{System Model AND IQML METHOD}

\section{A. System Model}

Assume that $D$ narrowband plane waves imping on an uniform linear array (ULA) equipped with $M$ antenna elements. At time instant $n$, the multi-dimensional received vector $\mathbf{y}(n)$ of size $M \times 1$, i.e., $\mathbf{y}(n)=\left[y_{1}(n), y_{2}(n), \ldots, y_{M}(n)\right]^{T}$, can be modelled as

$$
\mathbf{y}(n)=\mathbf{A x}(n)+\mathbf{w}(n)
$$


where $\mathbf{x}(n)=\left[x_{1}(n), x_{2}(n), \ldots, x_{D}(n)\right]^{T}$ is a $D \times 1$ source vector, $T$ being the transpose operator, and $\mathbf{w}(n)$ is an $M \times 1$ noise vector with independent zero-mean Gaussian random components and covariance matrix $\mathbf{K}_{w}=\sigma_{w}^{2} \mathbf{I}$. Matrix $\mathbf{A}$ denotes the array manifold matrix with $D$ array manifold vectors $\mathbf{a}\left(\theta_{i}\right), i=1,2, \cdots, D$,

$$
\mathbf{A}=\left[\mathbf{a}\left(\theta_{1}\right), \mathbf{a}\left(\theta_{2}\right), \cdots, \mathbf{a}\left(\theta_{D}\right)\right],
$$

where

$$
\mathbf{a}\left(\theta_{i}\right)=\left[1, e^{j \pi \sin \left(\theta_{i}\right)}, \cdots, e^{j \pi(M-1) \sin \left(\theta_{i}\right)}\right]^{T}
$$

and $\theta_{i}$ is the DOA associated with the $i$ th impinging plane wave.

Stacking up $N_{s}$ consecutive independent received vectors and defining $\mathbf{Y} \stackrel{\text { def }}{=}\left[\mathbf{y}(1), \cdots, \mathbf{y}\left(N_{s}\right)\right], \mathbf{X} \stackrel{\text { def }}{=}[\mathbf{x}(1), \cdots$, $\left.\mathbf{x}\left(N_{s}\right)\right]$, and $\mathbf{W} \stackrel{\text { def }}{=}\left[\mathbf{w}(1), \cdots, \mathbf{w}\left(N_{s}\right)\right]$, we obtain

$$
\mathbf{Y}=\mathbf{A X}+\mathbf{W}
$$

As $\mathbf{W}$ is Gaussian distributed, the conditional ML function can be written as

$$
\begin{aligned}
& f(\mathbf{Y} \mid \mathbf{X}, \mathbf{A})=\prod_{n=1}^{N_{s}} f(\mathbf{y}(n) \mid \mathbf{x}(n), \mathbf{A}) \\
& =\frac{1}{\pi^{M N_{s}}\left|K_{w}\right|^{N_{s}}} e^{-\sum_{n=1}^{N_{s}}\|\mathbf{y}(n)-\mathbf{A x}(n)\|_{\mathbf{K}_{w}^{-1}}^{2}},
\end{aligned}
$$

where

$$
\|\mathbf{x}\|_{\mathbf{K}^{-1}}^{2} \stackrel{\text { def }}{=} \mathbf{x}^{H} \mathbf{K}^{-1} \mathbf{x}
$$

denotes the generalized 2-norm of $\mathbf{x}$, and $H$ denotes the conjugate transpose. From (5), the conditional ML estimate can be written as

$$
\max _{\left\{\theta_{i}, \mathbf{x}\right\}}\left(-\sum_{n=1}^{N_{s}}\|\mathbf{y}(n)-\mathbf{A} \mathbf{x}(n)\|_{2}^{2}\right) .
$$

The solution, though can be found by exhaustic search, is computational intractable, especially when the array size $M$ and data block $N_{s}$ are large. An alternative scheme is to substitute the source vector $\mathbf{x}(n)$ by its least squared solution for any fixed $\mathbf{A}$, and transform (7) into

$$
\begin{aligned}
& \min _{\left\{\theta_{i}\right\}} \sum_{n=1}^{N_{s}}\left\|\mathbf{y}(n)-\mathbf{A A}^{\dagger} \mathbf{y}(n)\right\|_{2}^{2} \\
= & \min _{\left\{\theta_{i}\right\}} \sum_{n=1}^{N_{s}} \mathbf{y}^{H}(n) \mathbf{P}_{\mathbf{A}}^{\perp} \mathbf{y}(n) \\
= & \min _{\left\{\theta_{i}\right\}} N_{s} \operatorname{tr}\left(\mathbf{P}_{\mathbf{A}}^{\perp} \hat{\mathbf{R}}_{\mathbf{y}}\right),
\end{aligned}
$$

where $\dagger$ denotes the matrix pseudoinverse operation, $\perp$ the orthogonal projection, $\mathbf{P}_{\mathbf{A}}^{\perp} \stackrel{\text { def }}{=} \mathbf{I}-\mathbf{A} \mathbf{A}^{\dagger}$, and $\hat{\mathbf{R}}_{\mathbf{y}} \stackrel{\text { def }}{=} \frac{1}{N_{s}} \mathbf{Y} \mathbf{Y}^{H}$ is the sample correlation matrix.

\section{B. IQML method}

Using the coefficients of the polynomial

$$
b(z) \stackrel{\text { def }}{=} \prod_{i=1}^{D}\left(z-z_{i}\right)=b_{0}^{*}+b_{1}^{*} z+\ldots+b_{D}^{*} z^{D}
$$

where $\left\{z_{i} \stackrel{\text { def }}{=} e^{j \pi \sin \left(\theta_{i}\right)}\right\}$ are its roots and $*$ represents complex conjugate, Bresler and Macowski [2] construct the $M \times(M-D)$ Toeplitz matrix $\mathbf{B}$

$$
\mathbf{B}=\left[\begin{array}{cccc}
b_{0} & & & \\
\vdots & b_{0} & & \\
b_{D} & \vdots & \ddots & \\
& b_{D} & \ddots & b_{0} \\
& & \ddots & \vdots \\
& & & b_{D}
\end{array}\right] .
$$

They further proved that $\mathbf{P}_{\mathbf{A}}^{\perp}=\mathbf{P}_{\mathbf{B}}$, and (8) is equivalent to

$$
\begin{aligned}
& \min _{b} \mathbf{b}^{H}\left(\sum_{n=1}^{N_{s}} \mathbf{Y}^{T}(n)\left(\mathbf{B}^{T} \mathbf{B}^{*}\right)^{-1} \mathbf{Y}^{*}(n)\right) \mathbf{b} \\
& \stackrel{\text { def }}{=} \min _{b} \mathbf{b}^{H} \mathbf{Q b} \\
& \text { subject to: } b_{0}=1,
\end{aligned}
$$

where $\mathbf{b}=\left[b_{0}, b_{1}, \ldots, b_{D}\right]^{T}$ and $\mathbf{Y}(n)$ is the accumulated data matrix

$\mathbf{Y}(n) \stackrel{\text { def }}{=}\left[\begin{array}{cccc}y_{D+1}(n) & \cdots & y_{2}(n) & y_{1}(n) \\ \vdots & \ddots & \vdots & \vdots \\ y_{M}(n) & \cdots & y_{M-D+1}(n) & y_{M-D}(n)\end{array}\right]$.

The constraint (13) is imposed to guarantee a non-trivial $\mathbf{b}$. Instead of optimizing (12) directly, [2] searches the solution by iteratively projecting on the temporary solution space.

\section{Cross-Entropy Method}

For convenience of reference, we give a brief review of Rubinstein's CE method. Let $\Theta$ be the domain of variable $\theta$, and $S$ be the score function of $\theta$ defined on $\Theta$. The CE method attempts to solve the following optimization function

$$
\arg \max _{\theta \in \Theta} S(\theta)
$$

by relating the above problem to a rare event simulation problem. A popular approach to reduce the required sample size for estimating the rare event probability is the so-called importance sampling technique. To find the optimal importance sampling density within a class of densities $f(\theta ; \mathbf{v})$, one iteratively adapts the parameter $\mathbf{v}$ so that the Kullback-Leibler distance (cross entropy) between the associated density and the optimal importance sampling density is minimized. In short, a generic CE method can be described by the following two steps [3]. 
1. Generate samples from the importance density specified by the parameters from the previous iteration.

2. Update the parameters for next iteration according to the order of the score values associated with new samples and the CE criterion.

\section{ML DOA ESTIMATION BASED ON CE METHOD}

The score function of (7) is the total sum of the negative 2-norm of the error vectors. To apply the CE method, we have to include a set of important density functions over the deterministic $\mathbf{A}$ and $\mathbf{X}$. At the $k$ th iteration, for BPSK signals, a natural choice of density function for each element $\left\{\left\{x_{i}^{(k)}(n)\right\}_{i=1}^{D}\right\}_{n=1}^{N_{s}}$ in $\mathbf{X}^{(k)}$ is the Bernoulli distribution, i.e., $x_{i}^{(k)}(n) \sim \operatorname{Ber}\left(p_{i, n}^{(k)}\right)$, such that $P\left(x_{i}^{(k)}(n)=1\right)=1-$ $P\left(x_{i}^{(k)}(n)=-1\right)=p_{i, n}^{(k)}$.

Besides, we have to model the $D$-dimensional DOA information in the continuous space $[-\pi, \pi)$ for the manifold matrix $\mathbf{A}^{(k)}$. To be compatible with the results for spatially distributed source [4][6], each element $\left\{\sin \left(\theta_{i}^{(k)}\right)\right\}_{i=1}^{D}$ is modelled as truncated Gaussian distributed, i.e.,

$$
\sin \left(\theta_{i}^{(k)}\right) \sim \mathcal{N}\left(\mu_{i}^{(k)}, \sigma_{i}^{(k)}\right)
$$

given the constraint

$$
-1 \leq \sin \left(\theta_{i}^{(k)}\right) \leq 1, \text { for } i=1,2, \ldots, D .
$$

In order to sample $\left\{\sin \left(\theta_{i}^{(k)}\right)\right\}_{i=1}^{D}$ in the constraint region, we adopt the acceptance-rejection method described in [7]. By defining the approximated important density, the CE-ML algorithm for DOA estimation is described in Table I in which the following three recursive relations are used.

$$
\begin{aligned}
& p_{i, n}^{(k+1)}=\alpha \cdot \frac{1}{\sum_{u=1}^{U} \mathbf{I}_{\left\{S\left(\mathbf{X}_{u}^{(k)}, \mathbf{A}_{u}^{(k)}\right) \geq \gamma^{(k)}\right\}}} \\
& \cdot \sum_{u=1}^{U}\left[\mathbf{I}_{\left\{S\left(\mathbf{X}_{u}^{(k)}, \mathbf{A}_{u}^{(k)}\right) \geq \gamma^{(k)}\right\}} \mathbf{I}_{\left\{x_{i, u}^{(k)}(n)=1\right\}}\right] \\
& +(1-\alpha) p_{i, n}^{(k)} \\
& \mu_{i}^{(k+1)}=\alpha \cdot \frac{1}{\sum_{u=1}^{U} \mathbf{I}_{\left\{S\left(\mathbf{X}_{u}^{(k)}, \mathbf{A}_{u}^{(k)}\right) \geq \gamma^{(k)}\right\}}} \\
& \cdot \sum_{u=1}^{U}\left[\mathbf{I}_{\left\{S\left(\mathbf{X}_{u}^{(k)}, \mathbf{A}_{u}^{(k)}\right) \geq \gamma^{(k)}\right\}} \sin \left(\theta_{i, u}^{(k)}\right)\right] \\
& +(1-\alpha) \mu_{i}^{(k)} \\
& \left(\sigma_{i}^{(k+1)}\right)^{2}=\alpha \cdot \frac{1}{\sum_{u=1}^{U} \mathbf{I}_{\left\{S\left(\mathbf{X}_{u}^{(k)}, \mathbf{A}_{u}^{(k)}\right) \geq \gamma^{(k)}\right\}}-1} \\
& \sum_{u=1}^{U}\left[\mathbf{I}_{\left\{S\left(\mathbf{X}_{u}^{(k)}, \mathbf{A}_{u}^{(k)}\right) \geq \gamma^{(k)}\right\}}\left(\sin \left(\theta_{i, u}^{(k)}\right)-\mu_{i}^{(k+1)}\right)^{2}\right. \\
& +(1-\alpha)\left(\sigma_{i}^{(k)}\right)^{2}
\end{aligned}
$$

While the algorithm iterates, the generated standard deviation $\sigma_{i}^{(k)}$ decreases to zero. When the algorithm converges, the approximated important density degenerates to the impulse function, i.e. $\mathcal{N}\left(\theta_{i}^{*}, 0\right)$, matching the important density of the deterministic case in (7).

By sampling from the exact signal space, we obtain performance superior to that of the conventional covariance matrix based methods, which are corrupted by unknown signal phase. However, when the data block is large, sampling over such a high dimensional signal space of $\mathbf{X}(n)$ becomes impractical.

\section{CE-PPML METHOD}

To ease the computation burden of the pure CE method given in section IV, we propose an alternative $\mathrm{CE}$ method that does not generate samples from high dimensional $\mathbf{X}(n)$. We use the information generated from least square projection of the sample covariance matrix $\hat{\mathbf{R}}_{\mathbf{y}}$ in (9). This approximation greatly reduces the sampling complexity especially when the block size, $N_{s}$, is much larger than the number of impinging plane waves, $D$. Furthermore, we invoke the technique of polynomial parameterization described in Section II-B to simplify the calculation of $\mathbf{P}_{\mathbf{B}}=\mathbf{P}_{\mathbf{A}}^{\perp}$ in (9). Since the matrix $\left(\mathbf{B}^{H} \mathbf{B}\right)$ is a banded Hermitian matrix, its inverse can be efficiently computed via the inverse of a $D \times D$ matrix rather than an $(M-D) \times(M-D)$ matrix [8]. Although the required inverse matrix size $D \times D$ is the same as $\mathbf{P}_{\mathbf{A}}^{\perp}$, we can avoid calculating the cumbersome $(M-1) D$ exponential functions in $\mathbf{A}$ after applying polynomial parameterization, especially when the array size $M$ and the sample size $U$ are large. The resulting CE-PPML algorithm is given in Table II.

As the DOA information of the polynomial parameterization scheme is represented by $\mathbf{b}$, the proposed CE-PPML algorithm first generates a list of samples $\left\{\mathbf{b}_{u}\right\}_{u=1}^{U}$ from an auxiliary phase distribution. Following the basic framework of the CE method, we then replace the iterative projecting steps of the IQML algorithm by the iterative important sampling procedure in Table I. As will be proved by simulation, the divergence phenomenon of the conventional IQML method is greatly reduced by this method especially in low SNR region.

The main ideas of the proposed CE algorithm can be summarized by the two design criteria: (i) minimizing the number of target parameters by using deterministic signal processing techniques with performance and complexity constraints; and (ii) randomizing the desired parameters and applying the $\mathrm{CE}$ method. By following the criteria we are able to take the advantage of the global searching ability of the CE method while maintaining linear computing complexity.

\section{Vi. Simulation Results}

We consider a standard ULA equipped with $M=10$ antenna elements. Two adjacent elements of the ULA are separated by half wavelength. A single signal source with impinging arrival angle equal to $\sin (\theta)=-0.3$ is assumed. The block number $N_{s}$ is 10 . The sample size used in CEbased methods is 500 for all SNR regions and, except for Fig. 3, the smooth parameter $\alpha$ is 0.6. Maximum iteration 
number is set to be 200 . When the iteration number exceeds 200 , the algorithm is regarded as being fail to converge.

Fig. 1 shows the root mean squared error (RMSE) performance of the IQML and the proposed (CE-ML, and CEPPML) algorithms using a short block size. The stopping criterion used in Table I and Table II is based on the convergence of the score value in each iteration. The algorithms terminate when the difference of the score values of five successive iterations are within a predefined threshold, say $\eta=10^{-8}$. It is clearly that the two proposed CE-based methods outperform the IQML algorithm and the CE-ML algorithm yields the best overall average performance. The Cramér-Rao bounds for array systems with unknown complex and real signal source using the conditional ML approach [5], [9]

$$
\begin{aligned}
\operatorname{CRB}_{\text {complex }}(\psi) & =\frac{6 \cdot \mathrm{SNR}^{-1}}{N_{s}\left(M^{2}-1\right) M \pi^{2}}, \psi=\sin \theta \\
\operatorname{CRB}_{\text {real }}(\psi) & =\frac{3 \cdot \mathrm{SNR}^{-1}}{N_{s}(M-1) M(2 M-1) \pi^{2}}
\end{aligned}
$$

are also included in the figure for comparison purpose.

Since signal sources are estimated by the sample correlation matrix $\hat{\mathbf{R}}_{\mathbf{y}}$, the IQML and the proposed CE-PPML methods can only achieve the bound for unknown phase (complex) signal sources. On the other hand, the CE-ML method, having drawn samples from the signal domain thus avoiding possible phase error, can achieve the bound for known phase (real) signal sources. This performance advantage is obtained at the expense of increasing the required sample size for large data blocks. In other words, when $N_{s}$ is small, since the sample covariance matrix $\hat{\mathbf{R}}_{\mathbf{y}}$ is not good approximation of its ensemble counterpart, the proposed CE-ML method gives better performance than the covariance matrix based methods.

The large block size $\left(N_{s}=100\right)$ RMSE performance of the CE-PPML and IQML algorithms are depicted in Fig. 2. Other algorithmic parameters used are the same as the previous example. By using a larger data block size, the sample covariance matrix $\hat{\mathbf{R}}_{\mathbf{y}}$ becomes a closer approximation of the ensemble covariance matrix $\mathbf{R}_{\mathbf{y}}$ and the performance of both algorithms improves accordingly. The CE-PPML algorithm still outperforms the IQML method for $-10 \leq \mathrm{SNR} \leq 10$ dB. The convergence behavior comparison is given in Fig. 3 . Fig. 3 indicates that the average iteration numbers for the two proposed $\mathrm{CE}$ methods remain relatively low while the IQML solution tends to diverge in the low SNR region.

The computational complexity of the CE-based algorithm is proportional to the product of iteration number and sample size while that of the IQML algorithm is proportional to the iteration number. Nevertheless, the required iteration number of the latter is much larger than that of the former algorithms in low SNR region. Furthermore, samples used in CE-based algorithms can be generated in parallel and then the processing delay will mainly depend on the iteration number.

\section{CONCLUSION}

We present two CE-based methods to perform the maximum likelihood DOA estimation. Our first CE-based approach searches the ML solution in the full signal and angle space. It gives accurate DOA estimation even if the block size $N_{s}$ is small. However, when $N_{s}$ is large, the algorithm is computational infeasible. To make the CE-based solution practical, we introduce the covariance matrix approximation and the polynomial parameterization scheme in our algorithm. The resulting CE-PPML algorithm alleviates the error propagation arose from the iterative projection process in the IQML approach and is much more robust in the low SNR region.

Although we consider only the case when the impinging plane waves have deterministic DOA, the proposed method can be easily extended to solve DOA estimation problems for spatially distributed source with arbitrary constellation by including additional system parameters, e.g., angular spread.

\section{REFERENCES}

[1] H. L. Van Trees, Optimum array processing, Wiley, New York, 2002.

[2] Y. Bresler and A. Macovski, "Exact maximum likelihood parameter estimation of superimposed exponential signals in noise," IEEE Trans. ASSP., pp. 1081-1089, Oct. 1986.

[3] R. Y. Rubinstein and D. P. Kroese, The Cross-Entropy Method, Springer, 2004.

[4] O. Besson and P. Stoica, "Decoupled Estimation of DOA and Angular Spread for a spatially Distributed Source," IEEE Trans. Signal Process., July 2000.

[5] P. Stoica and A. Nehorai, "MUSIC, maximum likelihood, and CramérRao bound," IEEE Trans. ASSP., pp. 720-741, May 1989.

[6] J. B. Anderson, "Distributions of phase derivative in mobile communications," IEE Proc.-H, pp. 197-201, Aug. 1990.

[7] G. S. Fishman, Monte Carlo : Concepts, Algorithms, and Applications, Springer 1995.

[8] R. Kumaresan, et al., "An algorithm for pole-zero modelling and spectral analysis,” IEEE Trans. ASSP., pp. 637-640, June 1986.

[9] J. Li and R. T. Compton, "Maximum Likelihood Angle Estimation for Signals with Known Waveforms,” IEEE Trans. Signal Process., pp. 28502862, July 1993

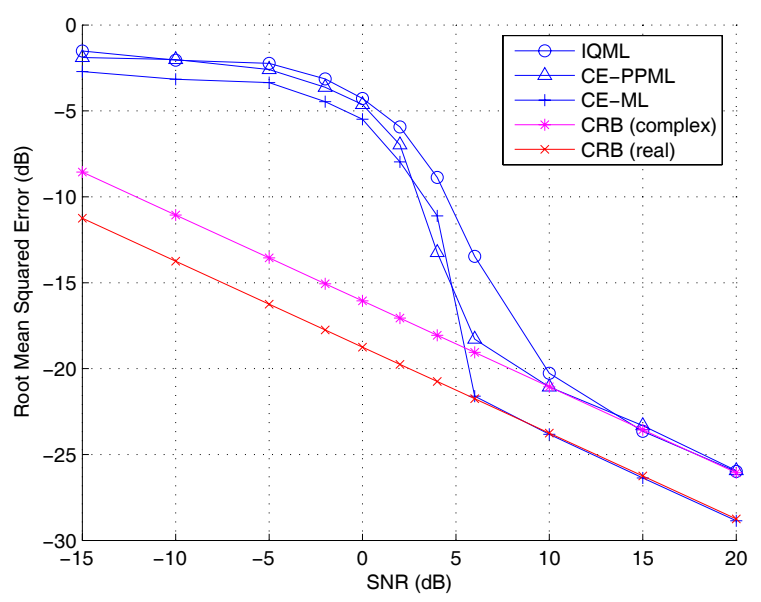

Fig. 1. Root Mean squared error (MSE) versus SNR: $M=10, N_{s}=$ $10, D=1, \alpha=0.6$, Trials $=500$. The DOA is set at $\sin (\theta)=-0.3$. 
TABLE I

CE METHOD FOR MAXIMUM LIKELIHOOD DOA ESTIMATION

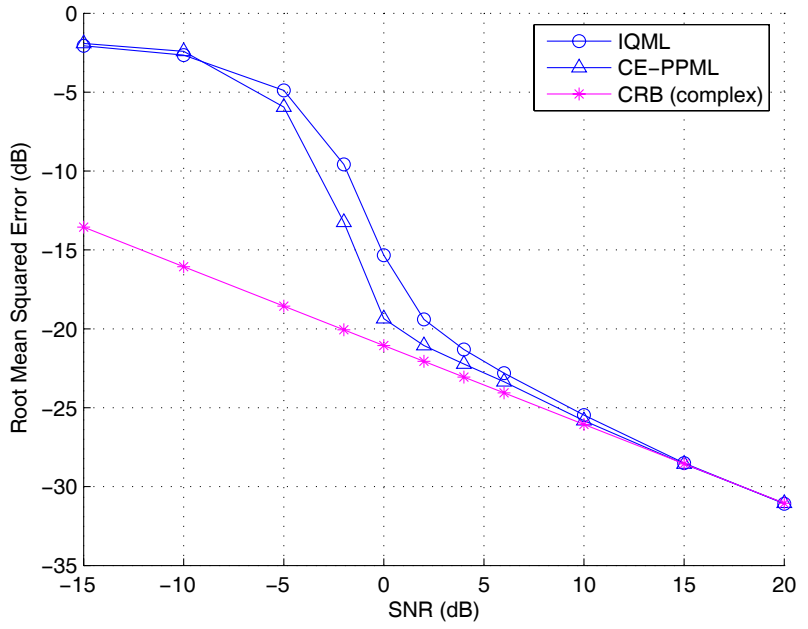

Fig. 2. Root Mean squared error (MSE) performance versus SNR: $M=$ $10, N_{s}=100, D=1, \alpha=0.6$, Trials $=1000$. The true DOA is at $\sin (\theta)=-0.3$

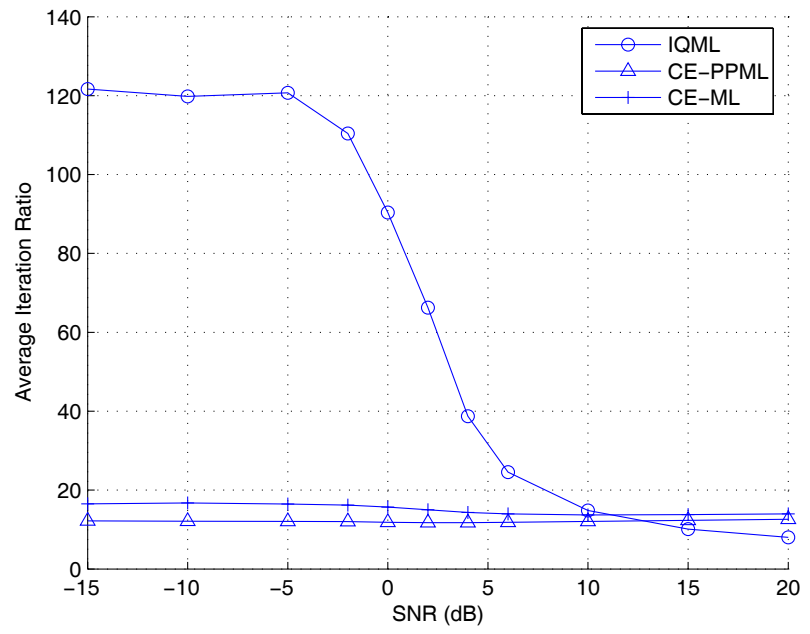

Fig. 3. Average Iteration Ratio (AIR) behavior as a function SNR: $M=$ 10, $N_{s}=10, D=1, \alpha=0.9$, Trial $=1000$. AIR is defined as $\frac{\text { total number of iterations }}{\text { total number of trials }}$. Hence, a higher AIR implies a lower convergence speed.
1. Initialize $p_{i, n}^{(0)}=0.5, \mu_{i}^{(0)}=0$, and $\sigma_{i}^{(0)}=1$. Set the quantile parameter $\rho$, and define a smooth parameter $0<\alpha<1$.

2. Draw $U$ samples $x_{i, u}^{(k)}(n) \sim \operatorname{Ber}\left(p_{i, n}^{(k)}\right)$. Draw $U$ samples $\sin \left(\theta_{i, u}^{(k)}\right) \sim \mathcal{N}\left(\mu_{i, u}^{(k)}, \sigma_{i}^{(k)}\right)$ by rejecting the samples that are outside the range $[-1,1]$. Construct $\left\{\mathbf{X}_{u}^{(k)}\right\}_{u=1}^{U}$, and $\left\{\mathbf{A}_{u}^{(k)}\right\}_{u=1}^{U}$ from the generated samples.

3. Calculate the score functions $\left\{S\left(\mathbf{X}_{u}^{(k)}, \mathbf{A}_{u}^{(k)}\right)\right\}$ according to (7).

4. Set a quantile parameter $\rho$, such that there is a $\gamma^{(k)}$ satisfying $\gamma^{(k)}=\arg \max _{\gamma} P(S(\mathbf{Z}) \geq \gamma) \geq \rho$ for $\mathbf{Z} \in\left\{\mathbf{X}_{u}^{(k)}, \mathbf{A}_{u}^{(k)}\right\}_{u=1}^{U}$.

5. Update the estimated Bernoulli parameter $p_{i, n}^{(k+1)}$ for $x_{i}^{(k+1)}(n)$ based on (17).

6. Stop at iteration $k=K$ if the pre-defined stopping criterion is met. Output $\left\{\mu_{i}^{(K+1)}\right\}_{i=1}^{D}$ as the estimate of $\left\{\sin \left(\theta_{i}\right)\right\}_{i=1}^{D}$. Otherwise, let $k=k+1$, update $\mu_{i}^{(k+1)},\left(\sigma_{i}^{(k+1)}\right)^{2}$ using (18), (19) and repeat Steps $2-4$.

TABLE II

CE-PPML ALGORITHM

1. Initialize $\mu_{i}^{(0)}=0$, and $\sigma_{i}^{(0)}=1$. Set the quantile parameter $\rho$, and a smooth parameter $0<\alpha<1$.

2. Draw $U$ samples $\sin \left(\theta_{i, u}^{(k)}\right) \sim \mathcal{N}\left(\mu_{i, u}^{(k)}, \sigma_{i}^{(k)}\right)$. Reject samples outside the range $[-1,1]$. Find $\mathbf{b}_{u}^{(k)}$ and construct the corresponding $\mathbf{B}_{u}^{(k)}$ described in (11) from the generated samples.

3. Calculate the score function

$$
\left\{S\left(\mathbf{b}_{u}^{(k)}\right) \stackrel{\text { def }}{=} N_{s} \operatorname{tr}\left(\mathbf{P}_{\mathbf{B}_{u}}^{(k)} \hat{\mathbf{R}}_{\mathbf{y}}\right)\right\}_{u=1}^{U}
$$

where $\mathbf{P}_{\mathbf{B}_{u}}^{(k)} \stackrel{\text { def }}{=} \mathbf{B}_{u}^{(k)}\left(\mathbf{B}_{u}^{(k)}{ }^{H} \mathbf{B}_{u}^{(k)}\right)^{-1} \mathbf{B}_{u}^{(k)}{ }^{H}$.

4. Set a quantile parameter $\rho$, such that there is a $\gamma^{(k)}$ satisfying

$$
\gamma^{(k)}=\arg \max _{\gamma} P(S(\mathbf{Z}) \geq \gamma) \geq \rho
$$

for $\mathbf{Z} \in\left\{\mathbf{b}_{u}^{(k)}\right\}_{u=1}^{U}$

5. Generate the estimated Gaussian parameter $\mu_{i}^{(k+1)}$ and $\sigma_{i}^{(k+1)}$ for next iteration according to (18) and (19) with $\left\{\mathbf{X}_{u}^{(k)}, \mathbf{A}_{u}^{(k)}\right\}$ being replaced by $\left\{\mathbf{b}_{u}^{(k)}\right\}$.

6. Stop at iteration $k=K$ if the pre-defined stopping criterion is met. Output $\left\{\mu_{i}^{(K+1)}\right\}_{i=1}^{D}$ as the estimate of $\left\{\sin \left(\theta_{i}\right)\right\}_{i=1}^{D}$. Otherwise, set $k=k+1$, and repeat Step 2 to Step 5. 